\title{
Interactive comment on "Sediment characteristics as an important factor for revealing carbon storage in Zostera marina meadows: a comparison of four European areas" by Martin Dahl et al.
}

\section{Anonymous Referee \#2}

Received and published: 8 June 2016

This study provides new insights into the organic carbon storage of Zostera meadows in Europe. The dataset compiled may constitute a good overview of the variability in carbon storage within and among sites for this seagrass species. The sites sampled have different habitat characteristics (e.g. geomorphologies, salinity, hydrodynamic energy, water depth, tides, species composition, etc.) and such factors play a role in sediment carbon storage while adding confounding factors and complex interactions between factors that need to be accounted for in the discussion of the manuscript. However, the low number of proxies analysed in the cores precludes such detailed approach, overall leading to relatively vague and well known conclusions: 'carbon storage 
in Zostera meadows is influenced by sediment grain-size, high porosity and low density of sediments'.

This manuscript would greatly benefit from the analysis of other proxies in the cores, such as tracers of organic matter origin (e.g. stable $\mathrm{C}$ and $\mathrm{N}$ isotopes), geomorphological study of the areas to determine whether the Corg stock is derived from the capacity of the plants to trap sediments or simply due to the depositional environment (e.g. fetch, share bottom stress), and dating of the sediments (14C or $210 \mathrm{~Pb}$ ) to determine whether the sediments are mixed (subjected to erosion which may explain lower Corg storage) and to compare 'apples with apples' in terms of period of accumulation rather sediment thickness.

I consider that this manuscript is not suitable for publication in its present form, and a major overhaul together with additional analyses are required prior to publication. Some of the concerns raised below (e.g. corrections for soil compression) most probably could not be addressed at this stage, which could compromise the reliability of the conclusions derived. l've also provided some tips to improve the manuscript (i.e. to give it a broader perspective), but I am not sure if after all the manuscript could meet the standards of the journal.

General comments: 1. The methods used for sediment grain-size (e.g. removing coarse plants and other grains that where there and contributed to the accumulation/preservation of Corg, use of $\mathrm{H} 2 \mathrm{O} 2$ or not, hydrometer vs sieving, etc.) are multiple and probably led to different results with important implications for the conclusions of the paper. It is important to demonstrate that grain-size results are reproducible between methods, since grain-size is the main driver detected in this study. Please discuss the potential implications of using different methods for sediment grain-size analyses, including the hydrometer.

2. Compression of loose soils could be as much as $30 \%$ and differ between sites, in particular between sites with different sediment grain-size composition. The authors

Printer-friendly version

Discussion paper 
did not account for sediment compression when estimating the Corg stocks for a certain depth, and therefore, this could be a very important but undesired factor explaining part of the conclusions reached by the authors. In other words, meadows in finer sediments most probably experienced higher compression than those growing in coarse sediment. As a result, an overestimation of the Corg content in meadows with finer sediments (more Corg in less space) could led to spurious differences between sites and meadow/bare, in particular when assessing differences in $\mathrm{g}$ Corg $\mathrm{cm}-2$. The fact that the total length of sample recovered during coring differed between sites (i.e. while using $50 \mathrm{~cm}$ long pipes in all cases) could evidence such issue.

3. This study fails to address whether the Corg storage in the meadows is due to the meadow itself (authochtonous inputs) or if it is mainly driven by the depositional environment (allochtonous inputs). In other words, would the Corg stocks be there independently of the presence of the meadow? How much of the Corg stock is due to the grain-size and how much due to the inputs from the plant? Does the plant enhance sediment accumulation and Corg compared to bare sediments? The comparison with bare sediments could be a good approach to address this, but it is not clear whether control bare sediments are realistic controls? (if yes, most probably they should have seagrass meadows in there), or if al least they have similar grain-size (geomorphology/hydrology) so could be used as a reference?

4. The English needs edition in some parts of the manuscript, in particular the introduction and discussion.

5. Plant cover and biomass refer to present (sampling date), but it seems possible that meadow structure have not been constant over the last years or centuries, so over the ca. $40 \mathrm{~cm}$-thick period of accumulation estimated. The lack of a permanent gradient within each study site (such as water depth driving seagrass density and cover) precludes such approach, and the assumption of constant plant cover etc. over time is hard to believe, in particular considering the dynamics of Zostera meadows. Discuss the limitations of using such plant parameters and assumptions involved.

Printer-friendly version

Discussion paper 
6. The discussion of the importance of sediment grain-size in Corg accumulation and preservation is poor (e.g. include the concepts of selective and non-selective preservation in marine sediments). Important papers to look at (and references therein) to enrich the discussion: - Serrano et al 2015 Biogeoscience discussion_Key factors influencing carbon storage in Posidonia. - Lavery et al 2013_PlosOne_Seagrass carbon in Australia

7. What percentage of variability the PCA explained? It is meaningful? This information should be provided. The use of the PCA seems unnecessary.

8. The manuscript could benefit from some estimates of total Corg storage in Zostera meadows within each site, and maybe within Europe if the authors could argue that the sites sampled are representative of Zostera meadows elsewhere, so they captured the range of variability. Comparisons with Corg storage (and sediment characteristic) in other Zostera (USA) and other plants could also add interesting information to the manuscript.

Particular comments: L74: 'coarser' instead of 'courser'

L124-125: 'Total nitrogen (NT) was measured due to possible alteration of the nitrogen values when treated with $\mathrm{HCl}$ (Harris et al., 2001)' This sentence does not make sense, please clarify.

L137-138: Please specify how the samples were washed to remove $\mathrm{H} 2 \mathrm{O} 2$ and whether this could affect the grain size composition (wet sieving or decanting the supernatant?).

L149-150: provide the equations and explain the potential issues linked to the use of logarithmic regressions to extrapolate corg stocks: could led to over-/under-estimates of Corg stocks compared to cores long enough to estimate the stocks directly? Why not linear regression for example?

L152-L157: The use of General Linear Mixed Models is a more appropriate and robust analysis, including bare/zostera, etc. as fixed factors in the design, and study

Printer-friendly version

Discussion paper 
site as a random factor. Sediment depth also needs to be included as fixed factors because sediment depth is not independent. Test \%Corg, gCorg cm-3 and grain-size, separately.

L182: replace 'unaffected' by 'constant'

L228-230: Here and in the abstract there is a misleading message: high sediment porosity and low sediment density is the result of high Corg content, not vice-versa, unless you merge the concept of grain-size.

L230-231: Misleading message: as per the comment above, but also consider that fine and Corg-rich bare sediment could also constitute high natural carbon sinks. Therefore, the clue here is to assess seagrass inputs into the sedimentary Corg pool, rather than the Corg sink associated to the natural depositional environment. Data on e.g. stable $C$ and $\mathrm{N}$ isotopes of the organic matter that could provide an indication of the origin of the Corg stores within and among sites is missing, which largely constrains the conclusions derived from this study (see e.g. Serrano et al. 2016 Biogeosciences discussions_mud vs seagrass Corg for further details on the topic).

L249: the age of the sediments and associated sediment accumulation rates could also explain the differences found. Are we comparing $25 \mathrm{~cm}$-thick and $500 \mathrm{yr}$-old with $25 \mathrm{~cm}$-thick and $1000 \mathrm{yr}$ old Corg stocks? Discuss the implications

L259: reference submitted is missing in the list.

L254-256: This statement is misleading: from both studies the main message derived is that sediment grain-size has always an influence, but the amount of seagrass-detritus inputs into the sediment makes the difference breaking the linear relationship expected between fine sediments and bulk Corg content. Read the references cited carefully and clarify.

L257-267: This section of the discussion is poor: (i) the relationships between Corg, porosity and density have poor relevance (well know in soil science), in particular if

Printer-friendly version

Discussion paper 
they have not been corrected for compression during coring; (ii) cut this section to provide the main message: Corg content in Zostera meadows at Asko is lower due to the coarser sediments in which they inhabit.

\section{L277: 'was' instead of 'were'}

L285: add a comma

- The water depth gradient within and among sites is small, with differing tide regimes, etc. Is there any relationship between meadow structure and water depth that could provide more light into the impact of depth between sites?

Table 2: add the results of the ANOVA itself and some descriptives such as df and $\mathrm{F}$.

Figure 3: remove negative values from the $\mathrm{x}$-axis (set to zero), and place the graphs of Zostera and ungetated in the same plot for each site, reducing figure size and allowing to detect difference visually (the scale of the x-axis differ within and among sites, making interpretation difficult.

Table 4: include data on \%Corg and Corg density in this table.

Figure 4. Define $\mathrm{Bg}$ and $\mathrm{Ag}$ in the caption

Interactive comment on Biogeosciences Discuss., doi:10.5194/bg-2016-137, 2016. 